\title{
Integer Part of Cube Root and Its Combination
}

\section{Zhongguo Zhou}

College of Science, Hohai University, Nanjing, China

Email: zhgzhou@hhu.edu.cn

Received 6 July 2015; accepted 8 November 2015; published 11 November 2015

Copyright (C) 2015 by author and Scientific Research Publishing Inc.

This work is licensed under the Creative Commons Attribution International License (CC BY). http://creativecommons.org/licenses/by/4.0/

c) (i) Open Access

\section{Abstract}

For the cube root of a positive integer, a direct method to determine the floor of integer combination of the cube root and its square is given.

\section{Keywords}

\section{Cube Root, Integer Part, Continued Fraction}

\section{Introduction}

The continued fraction expansion of $\sqrt{2}$ can be calculated as follow(cf. [1]-[3]).

$$
\sqrt{2}=1+(\sqrt{2}-1)=1+\frac{1}{\sqrt{2}+1} \text {. }
$$

Because

$$
\sqrt{2}+1=2+(\sqrt{2}-1)
$$

hence

$$
\sqrt{2}=1+(\sqrt{2}-1)=1+\frac{1}{2+(\sqrt{2}-1)}=1+\frac{1}{2+\frac{1}{\sqrt{2}+1}} .
$$

When one calculates the continued fraction expansion of $\sqrt{2}$, it is important to determine the integer part of $m \sqrt{2}+n, m, n \in \mathbb{Z}$. For square root, its continued fraction expansion can be obtained easily because it is circled while there is no obvious method to do so for cube root. In this note, we will determine the integer part of cube root and its combination. So we can achieve the continued fraction expansion of cube root according to the in- 
teger part of the cube root.

\section{Main Results}

Let $N$ be a positive integer and not a cube. Denote $\alpha=\sqrt[3]{N}$ as its cube root. For $a \alpha^{2}+b \alpha+c, a, b, c \in \mathbb{Z}$, set

$$
p=b^{2}-a c, q=a^{2} N-b c, r=c^{2}-a b N, M=a^{3} N^{2}+b^{3} N+c^{3}-3 a b c N .
$$

Then these numbers are satisfied the identity:

$$
\left(a \alpha^{2}+b \alpha+c\right)\left(p \alpha^{2}+q \alpha+r\right)=M .
$$

We achieve two interesting properties on $a \alpha^{2}+b \alpha+c$ and $p \alpha^{2}+q \alpha+r$.

Theorem 1. If $a \geq 0$, then $p \alpha^{2}+q \alpha+r>0$. Therefore the number $a \alpha^{2}+b \alpha+c$ has the same sign as $M$. We consider two cases respectively.

1) If $b<0$.

a) If $c \geq 0$. Substituting $p=b^{2}-a c, q=a^{2} N-b c, r=c^{2}-a b N$ into the expression $p \alpha^{2}+q \alpha+r$, then we have

$$
\begin{aligned}
p \alpha^{2}+q \alpha+r & =a^{2} \alpha^{4}-a c \alpha^{2}+\frac{1}{4} c^{2}-a b \alpha^{3}+\frac{3}{4} c^{2}+b^{2} \alpha^{2}-b c \alpha \\
& =\left(a \alpha^{2}-\frac{1}{2} c\right)^{2}+\left(-a b \alpha^{3}\right)+\frac{3}{4} c^{2}+b^{2} \alpha^{2}+(-b c \alpha)>0
\end{aligned}
$$

since every term in the last expression is nonnegative.

b) If $c<0$. We have also the similar expression as case a):

$$
\begin{aligned}
p \alpha^{2}+q \alpha+r & =b^{2} \alpha^{2}-b c \alpha+\frac{1}{4} c^{2}+a^{2} \alpha^{4}-a c \alpha^{2}+\frac{3}{4} c^{2}-a b \alpha^{3} \\
& =\left(b \alpha-\frac{1}{2} c\right)^{2}+a^{2} \alpha^{4}+\left(-a c \alpha^{2}\right)+\frac{3}{4} c^{2}+\left(-a b \alpha^{3}\right)>0 .
\end{aligned}
$$

The above inequality holds because $a \geq 0, b<0, c<0$.

2) If $b \geq 0$.

a) If $c>-\sqrt{a b N}$. Then we have

We also have

$$
a \alpha^{2}+b \alpha+c>a \alpha^{2}+b \alpha-\sqrt{a b N} \geq 2 \sqrt{a \alpha^{2} b \alpha}-\sqrt{a b N} \geq \sqrt{a b N}>0 .
$$

$$
\begin{gathered}
a^{3} N^{2}+b^{3} N+c^{3}-3 a b c N>3 \sqrt[3]{a^{3} N^{2} b^{3} N c^{3}}-3 a b c N=0, \quad(c \geq 0) ; \\
a^{3} N^{2}+b^{3} N+c^{3}-3 a b c N \geq a^{3} N^{2}+b^{3} N+c^{3} \geq a^{3} N^{2}+b^{3} N-\sqrt{a^{3} b^{3} N^{3}}>0, \quad(-\sqrt{a b N}<c<0) .
\end{gathered}
$$

Hence by the identity (1)

$$
p \alpha^{2}+q \alpha+r=\frac{a^{3} N^{2}+b^{3} N+c^{3}-3 a b c N}{a \alpha^{2}+b \alpha+c}>0 .
$$

b) If $c \leq-\sqrt{a b N}$. Then $c^{2}>a b N, p=b^{2}-a c>0, q=a^{2} N-b c>0$. Because of $\alpha>1, \alpha^{2}>1$, therefore

$$
p \alpha^{2}+q \alpha+r>p+q+r=\left(b^{2}-a c\right)+\left(a^{2} N-b c\right)+\left(c^{2}-a b N\right)>0 .
$$

So we show that $p \alpha^{2}+q \alpha+r>0$ for both cases. According to identity (1), both $a \alpha^{2}+b \alpha+c$ and $M$ have the same sign.

Remark 1. The result is very amazing. Because the quotient ring $\mathbb{Q}[x] /\left(x^{3}-N\right) \simeq \mathbb{Q}(\alpha)$ is a filed, where $\left(x^{3}-N\right)$ is the maximal ideal generated by the irreducible polynomial $x^{3}-N$. For any $a \alpha^{2}+b \alpha+c \in \mathbb{Q}(\alpha)$, there exists $p \alpha^{2}+q \alpha+r$ such that

$$
\left(a \alpha^{2}+b \alpha+c\right)\left(p \alpha^{2}+q \alpha+r\right) \in \mathbb{Q}
$$


But it is surprising that the number $p \alpha^{2}+q \alpha+r$ defined in Theorem 1 is always positive.

Theorem 2. If $a^{3} N^{2}+b^{3} N+c^{3}-3 a b c N>0$ and $a^{3} N^{2}+b^{3} N+(c-1)^{3}-3 a b(c-1) N<0$, then

$$
\left[a \sqrt[3]{N^{2}}+b \sqrt[3]{N}\right]=-c
$$

That is to say, $-c$ is the biggest integer less than or equal to $a \sqrt[3]{N^{2}}+b \sqrt[3]{N}$.

According to Theorem 1,

$$
a \sqrt[3]{N^{2}}+b \sqrt[3]{N}+c>0, \quad a \sqrt[3]{N^{2}}+b \sqrt[3]{N}+c-1<0
$$

Hence

$$
-c<a \sqrt[3]{N^{2}}+b \sqrt[3]{N}<-c+1
$$

So

$$
\left[a \sqrt[3]{N^{2}}+b \sqrt[3]{N}\right]=-c
$$

The proof is completed.

Remark 2. Applying the Theorem 2, we can design an algorithm to calculate the continued fraction expansion of the cube root $\sqrt[3]{N}$.

\section{Acknowledgements}

The authors wish to thank Prof. Xiangqin Meng for her some helpful advices.

\section{References}

[1] Hardy, G.H. and Wright, E.M. (2008) An Introduction to the Theory of Numbers. 6th Edition, Oxford University Press, Oxford.

[2] Hensley, D. (2006) Continued Fractions. World Scientific.

[3] Karpenkov, O. (2013) Geometry of Continued Fractions. Springer-Verlag, Berlin. 